\title{
On the Longitudinal Stability of Cooled Coasting Ion Beams
}

\author{
Sergei Nagaitsev \\ Indiana University Cyclotron Facility \\ 2401 Milo B. Sampson Ln., Bloomington, IN 47405 USA
}

\section{Abstract}

The longitudinal stability of intense, low momentum spread ion beams is of great interest today, especially in electroncooled storage rings. Stability conditions are usually formulated as the limitations on the allowed value of beamchamber coupling impedances. In the case of cooled beams, the effect of cooling can not be neglected if the cooling rate is comparable with the revolution frequency spread. The effect of the electron cooling can be described by the Fokker-Planck equation. In this paper we present a simple analytical method to derive dispersion relations for the longitudinal coasting beam instabilities using a perturbative approach. Numerical examples applied to $45 \mathrm{MeV}$ protons in the IUCF cooling ring are presented.

\section{INTRODUCTION}

The interest in the longitudinal stability of the coasting cooled ion beams was stimulated by the number of publications [1], [2] reporting measured stability diagrams significantly different from what one could expect for the coasting beams with Gaussian momentum distributions in the absence of any cooling mechanism. In Ref. [3] two possible reasons of this difference were suggested: deviation of the momentum distribution from Gaussian and the influence of the electron cooling on the coherent stability of the ion beam. Both the non-Gaussian distributions and the influence of the cooling have been studied elsewhere [3], [4]. This paper elaborates on the derivation of dispersion relation in the presence of cooling in the closed analytical form. It is also oriented towards practical application for the IUCF cooler ring.

\section{DISPERSION RELATION}

\section{A. Fokker-Planck Equation}

The influence of the cooling on the particle distribution function $\psi$ can be described by the Fokker-Planck equation [4], [5]:

$$
\frac{\partial \psi}{\partial t}+\dot{\theta} \frac{\partial \psi}{\partial \theta}+\dot{\delta} \frac{\partial \psi}{\partial \delta}=\frac{\partial}{\partial \delta}\left(\lambda \delta \psi+\frac{D}{2} \frac{\partial \psi}{\partial \delta}\right)
$$

Here $\theta \equiv s /(2 \pi R)$ is the longitudinal coordinate, $\delta \equiv \Delta p / p$ is the conjugate momentum variable and $t$ is time. The cooling rate $\lambda$ and diffusion coefficient $D$ are assumed to be constant in (1). For coasting beams the stationary distribution $\psi_{0}(\delta)$ satisfies the equation:

$$
\lambda \delta \psi_{o}+\frac{D}{2} \frac{d \psi_{o}}{d \delta}=0
$$

The solution of (2) is the Gaussian distribution function:

$$
\psi_{0}(\delta)=\frac{N}{(2 \pi)^{1 / 2} \sigma} e^{-\delta^{2} / 2 \sigma^{2}}
$$

where $\sigma=(D / 2 \lambda)^{1 / 5}$ is the rms momentum spread of the beam and $N$ is a number of particles. One can now describe the perturbed particle distribution function as:

$$
\psi(\delta, \theta, t)=\psi_{o}(\delta)+\psi_{l}(\delta) e^{i(n \theta-\Delta t)}
$$

where $\psi_{1}$ is a small perturbation of the stationary distribution function, $n$ is a positive integer and $\Omega$ is the complex frequency of this coherent perturbation. Let us now assume that $\psi_{1}(\delta)$ is normalized to the number of particles involved in the perturbation:

$$
\int_{-\infty}^{+\infty} \psi_{l}(\delta) d \delta=N_{1}=\frac{2 \pi I_{1}}{e \omega_{o}}
$$

where $\omega_{o}$ is the revolution frequency of the synchronous particle and $e$ is the ion charge. Taking into account that

$$
\begin{gathered}
\dot{\theta}=\omega \\
\dot{\delta}=\frac{1}{\beta^{2}} \frac{\Delta \dot{E}}{E}=-\frac{e \omega_{o} I_{1} Z_{\|}}{2 \pi \beta^{2} E} e^{i(n \theta-o t)}
\end{gathered}
$$

where $Z_{\|}$is the longitudinal coupling impedance, $E$ is the energy of the beam and $\beta$ is the usual relativistic parameter, Eq. (1) can be rewritten to the first order as:

$$
i(n \omega-\Omega) \psi_{1}-\frac{d \psi_{0}}{d \delta} \frac{e \omega_{0} I_{1} Z_{\|}}{2 \pi \beta^{2} E}=\frac{d}{d \delta}\left(\lambda \delta \psi_{1}+\frac{D}{2} \frac{d \psi_{l}}{d \delta}\right)
$$

Here $\omega$ is the revolution frequency of the particle with momentum $\delta: \omega(\delta)=\omega_{o}(l-\eta \delta)$, where $\eta$ is the phase slip factor $\left(\eta \equiv-\Delta \omega / \omega_{o} \delta\right)$.

\section{B. Dispersion relation}

Since $\psi_{l}$ vanishes at the infinity, the natural way to solve 
equation (7) is by the Fourier transform method:

$$
f(k)=\frac{1}{\sqrt{2 \pi}} \int_{-\infty}^{+\infty} \psi_{1}(\delta) e^{i k \delta} d \delta
$$

which decreases the order of the differential equation (7) by one. The transformed equation can be solved by simple integration. Introducing the new notations:

$$
a_{n}=\frac{n \omega_{o}-\Omega}{\lambda} ; \quad q_{n}=\frac{n \eta \sigma \omega_{o}}{\lambda}
$$

the dispersion relation can be written as follows:

$$
1=i \frac{e I_{o}}{2 \pi \beta^{2} \sigma^{2} \eta E} \frac{Z_{1}}{n} \int_{0}^{q_{n}}\left(1-\frac{x}{q_{n}}\right)^{i a_{n}+q_{n}^{2}-1} e^{q_{n} x} x d x
$$

where $l_{o}$ is the beam current. Equation (10) can also be expressed in analytical form as [6]:

$$
1=i A \frac{Z_{1}}{n} q_{n}^{2} B\left(i a_{n}+q_{n}^{2}, 2\right) \Phi\left(2 ; i a_{n}+q_{n}^{2}+2 ; q_{n}^{2}\right)
$$

where $A$ is given by:

$$
A=\frac{e I_{o}}{2 \pi \beta^{2} \sigma^{2} \eta E}
$$

and $B(x, y), \Phi(\alpha ; \gamma ; z)$ are the beta function and the degenerate hypergeometric function respectively.

\section{Asymptotic Behavior}

In the case of vanishing cooling $(\lambda \rightarrow O)$ the dispersion relation (10) has to become the well-known dispersion relation for a Gaussian beam without cooling. In fact, recalling that the ratio $a_{n} / q_{n}$ remains finite, after some algebra one can write:

$$
1=i A \frac{Z_{1}}{n} J\left(\frac{\Omega-n \omega_{o}}{n \eta \sigma \omega_{o}}\right)
$$

where $J\left(u_{0}\right)$ is given by the expression:

$$
J\left(u_{0}\right) \equiv \int_{0}^{+\infty} e^{-x^{2} 2+i u_{0} x} x d x=\frac{1}{\sqrt{2 \pi}} \int_{-\infty}^{+\infty} \frac{u}{u-u_{0}} e^{-u^{2} / 2} d u
$$

Another limiting case is the coherent perturbation with a high harmonic number $(n \rightarrow \infty)$. This case is almost identical to the previous one. Cooling is too "slow" for such oscillations and the only stabilizing mechanism is Landau damping. For high harmonic numbers the dispersion relation reduces to (13).

\section{Stability Diagram}

Figure 1 shows the stability diagrams, calculated using Eq. 10, for the first three harmonics of a proton beam $(45 \mathrm{MeV})$ in the IUCF cooler ring. The cooling rate was taken to be $70 \mathrm{~Hz}$ [7] and momentum spread $4 \cdot 10^{-5}$. The stability diagram for the Gaussian beam, given by (13), is also shown on Fig. 1.

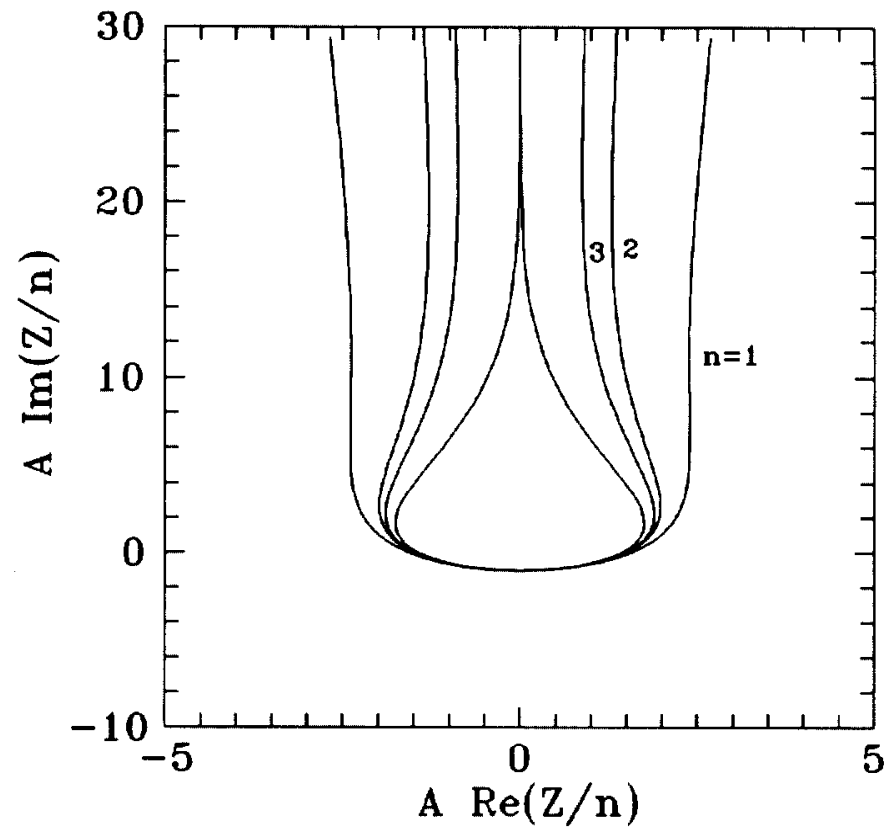

Figure 1. Variations of the stability diagram with harmonic number $\left(q_{1}=-2.5\right)$ for the cooled beam and stability diagram for the Gaussian beam without cooling.

\section{SCHOTTKY SPECTRUM}

The dielectric permeability function of the beam corresponding to the dispersion relation (10) can be written as:

$$
\varepsilon_{n}=1-i A \frac{Z_{1}}{n} \int_{0}^{q_{n}}\left(1-\frac{x}{q_{n}}\right)^{i a_{n}+q_{n}^{2}-1} e^{q_{n} x} x d x
$$

For a given $n$, equation (10) yields the frequency (generally complex) at which a perturbation can exist in the beam without an external source. For vanishing impedance or current the effect of polarization of the medium is negligible and we have $\varepsilon_{n}=1$. The Schottky noise power spectrum then can be written, using the dielectric function [1]:

$$
P_{n}(\Omega)=\frac{e^{2}}{\pi} \frac{N}{n} \frac{\Psi_{o}(\Omega / n)}{\left|\varepsilon_{n}\right|^{2}}
$$

The main feature of this Schottky spectrum is that at high 
beam intensities, it is strongly deformed into a double-peak shape [8], corresponding to the excitation of the slow and the fast coherent longitudinal waves by the external electric field or by beam current fluctuations. The frequency of this wave depends solely upon the beam current and the value of coupling impedance. Although it is generally a nontrivial problem to calculate the coupling impedance, one can make reasonable assumptions for the low energy electron-cooled rings. First, assume that $\left|\operatorname{Im} Z_{\mid} / n\right| \gg\left|\operatorname{Re} Z_{\|} / n\right|$ and second, that $Z_{\|} / n$ is independent of harmonic number $n$. These two assumptions correspond to the case of space-charge dominated impedance, given by:

$$
\frac{Z_{1}}{n}=-i \frac{Z_{o}}{2 \beta r^{2}}\left(2 \ln \left(\frac{b}{a}\right)+1\right)
$$

where $Z_{o}=377 \Omega, a$ and $b$ are diameters of the beam and vacuum chamber respectively. For the IUCF cooler ring at $45 \mathrm{MeV}$ expression (17) gives $\left|\operatorname{Im} Z_{\mid} / n\right| \approx 4 k \Omega$, whereas the real part of the impedance (resistive wall and broad-band) is typically less than $5 \Omega$ for any harmonic. Having made these assumptions and neglecting the momentum spread of the beam, we can obtain for the coherent frequencies in the absence of the cooling:

$$
\Delta \Omega=\Omega-n \omega_{0}= \pm n \omega_{0}\left|\frac{e \eta I_{0}}{2 \pi \beta^{2} E}\right|^{1 / 2}\left|\frac{Z_{1}}{n}\right|^{1 / 2}
$$

One can notice, that the value of $\Delta \Omega / n \omega_{0}$ is independent on $n$. This can be also seen directly from (13) after some algebraic transformations. For a cooled beam this is no longer true. In fact, the deviation of $\Delta \Omega / n \omega_{0}$ from a constant value for different harmonics is the easiest way to observe the influence of the cooling on coherent stability. Fig. 2 shows the frequency for coherent oscillations, given by (18) as well as the frequency in the presence of cooling for a $0.5 \mathrm{~mA}, 45$ $\mathrm{MeV}$ proton beam in the IUCF cooler ring.

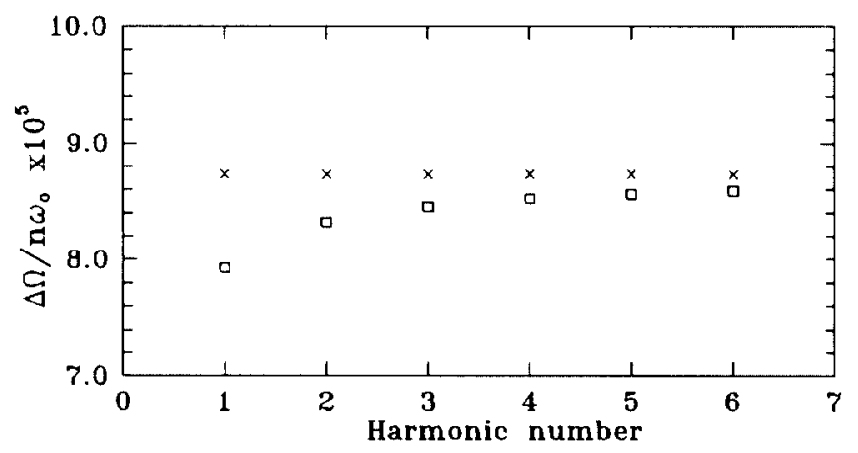

Figure 2. Frequency of coherent oscillations as a function of harmonic number for the beam with Gaussian momentum distribution in the absence ( $X^{\prime} s$ ) and in the presence ( ${ }^{\prime}$ 's) of cooling.

\section{CONCI,USION}

We have calculated the dispersion relation for cooled coasting beams. The influence of the cooling on the coherent beam stability can be observed by measuring the separation of the two coherent peaks in the Schottky spectrum as a function of harmonic number. The momentum distribution function of the beam can be deduced from the shape of the Schottky power spectrum using (16). Beam transfer function measurements [1] can be also performed to obtain stability diagrams directly.

\section{ACKNOWLEDGMENTS}

We would like to thank S.Y. Lee, D.V. Pestrikov and T. Ellison for useful discussions. We also would like to point out that the Fokker-Planck approach for the electron cooled beams was initially developed by D.V. Pestrikov in Ref. [4], [8].

\section{REFERENCES}

[1] I. Hofmann, "Beam dynamics of cooled heavy ion beams", Conference Record of the 1991 IEEE Particle Accelerator Conference, Vol. 4, pp. 2492-2496.

[2] J. Bosser et al., "Electron beam cooling and beam instability studies at LEAR", CERN/PS 92-45(AR), 1992.

[3] S. Cocher and I. Hofmann, "On the stability and diagnostics of heavy ions in storage rings with high phase-space density", Particle Accelerators, 1990, Vol. 34, pp. 189-210.

[4] D.V. Pestrikov, "The longitudinal Schottky noise of intense beam", Proceedings of the Workshop on Electron Cooling and New Cooling Techniques, Legnaro, Padova-Italy, 15-17 May, 1990, pp. 165-180

[5] H. Risken, "The Fokker-Planck Equation", 2nd edition, Springer-Verlag, 1989, p. 7.

[6] I.S. Gradshteyn and I.M. Ryzhik, "Table of Integrals, Series, and Products", New York: Academic Press, 1980 , p. 318 , formula 3.383 .1

[7] Tim Ellison, "Electron cooling", Ph.D. Thesis, Indiana University, 1990, p. 77.

[8] V.V. Parkhomchuk and D.V. Pestrikov, Sov. Phys. Tech. Phys., Vol. 25(7), p. 818 (1980). 